\title{
An improved 2b-RAD approach (I2b-RAD) offering genotyping tested by a rice (Oryza sativa L.) F2 population
}

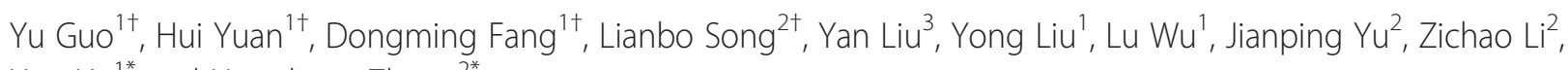
Xun $\mathrm{Xu}^{1^{*}}$ and Hongliang Zhang ${ }^{2^{*}}$

\begin{abstract}
Background: 2b-RAD (type IIB endonucleases restriction-site associated DNA) approach was invented by Wang in 2012 and proven as a simple and flexible method for genome-wide genotyping. However, there is still plenty of room for improvement for the existent 2b-RAD approach. Firstly, it doesn't include the samples pooling in library preparation as other reduced representation libraries. Secondly, the information of 2b-RAD tags, such as tags numbers and distributions, in most of species are unknown. The purposes of the research are to improve a new 2b-RAD approach which possesses samples pooling, moreover to figure out the characteristic and application potentiality of 2b-RAD tags by bioinformatics analysis.

Results: Twelve adapter1 and an adapter2 were designed. A library approach comprising digestion, ligation, pooling, PCR and size selection were established. For saving costs, we used non-phosphorylated adapters and indexed PCR primers. A F2 population of rice (Oryza sativa .L) was genotyped to validate the new approach. On average, 2000332 high quality reads of each sample were obtained with high evenness. Totally 3598 markers containing 3804 SNPs were discovered and the missing rate was 18.9\%. A genetic linkage map of 1385 markers was constructed and $92 \%$ of the markers' orders in the genetic map were in accordance with the orders in chromosomes. Meanwhile, the bioinformatics simulation in 20 species showed that the BsaXI had the most widespread recognition sites, indicating that 2b-RAD tags had a powerful application potentiality for high density genetic map. Using modified adapters with a fix base in $3^{\prime}$ end, 2b-RAD was also fit for QTL studies with low costs.
\end{abstract}

Conclusions: An improved 2b-RAD genotyping approach was established in this research and named as I2b-RAD. The method was a simple, fast, cost-effective and multiplex sequencing library approach. It could be adjusted by selecting different enzymes and adapters to fit for alternative uses including chromosomes assembly, QTL fine mapping and even natural population analysis.

Keywords: 2b-RAD, Genotyping, Genetic linkage map

\section{Background}

Genotyping with the molecular markers - detecting the heritable polymorphisms among the individuals of one or more populations - are employed in many regions in modern biological research including phylogeny, evolution, plant breeding and disease research, etc. [1-3].

\footnotetext{
*Correspondence: xuxun@genomics.cn; zhang|@cau.edu.cn

${ }^{\dagger}$ Equal contributors

'Beijing Genome Institute-Shenzhen, Beishan Industrial Zone, Yantian District, Shenzhen 518083, China

${ }^{2}$ Beijing Key Laboratory of Crop Genetic Improvement, College of Agronomy and Biotechnology, China Agricultural University, Beijing 100193, China Full list of author information is available at the end of the article
}

The classical molecular markers, such as restriction fragment length polymorphisms (RFLPs), randomly amplified polymorphic DNA (RAPD), amplified fragment length polymorphisms (AFLPs) and simple sequence repeats (SSRs) were proven to be powerful in genotyping. However, based on gel electrophoresis, these methods usually take long time and high labor costs with large samples size. In addition, the DNA polymorphisms with artificial bands counting are prone to error. Moreover, these methods generally produce limited markers, making it difficult to construct high density genetic map that is essential for chromosomes assembly. 
Therefore, the fast, cost-effective and high-throughput genotyping techniques are required.

Now, with the advent of next-generation sequencing (NGS), there are several such approaches, which are capable of genotyping not hundreds but thousands of markers in a single step [4]. Some newly developed techniques, combining NGS with restriction enzymes (REs) fragments, are widely used for genotyping. These techniques internally sequence the regions around REs recognition sites; produce a reduced representation of a genome. The restriction-site associated DNA (RAD) technique is capable of sequencing the regions adjacent to recognition site of a chosen restriction enzyme and widely applied to detecting single nucleotide polymorphisms (SNPs), even insertions/deletions (InDels), for quantitative trait locus (QTL) or evolutional analysis [5-8]. With a simple, quick library approach and lower coverage requirement, genotyping-by-sequencing (GBS) is particularly well suited for genotyping populations with large samples sizes [9-11]. Two-enzyme GBS and double digest RAD sequencing are the other two reduced representation libraries both based on simultaneous double REs digestion, which are also used for SNP discovery and genotyping [12,13].

The 2b-RAD (type IIB endonucleases restriction-site associated DNA) approach relies on the type IIB REs, such as BsaXI or AlfI (both insensitive enzyme for methylation), to produce uniform tags. It was proven to be a simple and flexible method for genome-wide genotyping [14]. Poland JA et.al compared many reduced representation libraries and concluded that $2 \mathrm{~b}-\mathrm{RAD}$ approach had the advantages of producing uniform length tags, allowing nearly all of the restriction sites to be surveyed and permitting marker intensity adjustment [15]. However, there is still plenty of room for improvement of the existent $2 \mathrm{~b}-\mathrm{RAD}$ approach. Firstly, it is not as multiplex as other reduced representation libraries using barcode adapter and pooling procedure. By using barcode adapters, many samples equal to one sample to carry on the subsequent library procedures after pooling. Therefore, it can save much time and labor cost. The RAD's multiplexing level achieves 96 samples while the GBS achieves 48 up to 384 , for instance [15]. The existent 2b-RAD approach achieves its multiplexing sequencing by using indexed PCR primers. The disadvantage is that each sample is prepared to a single sequencing library. It takes more times and labor cost, and is not fit for the populations with large sample size. Lacking of fast library approach would limit its application. Secondly, the information of 2b-RAD tags, such as tags numbers and distributions, in most of species are unknown except Arabidopsis thaliana [14]. In order to make better use of it, it is necessary to do a comprehensive testing.
Based on the above reasons, firstly, we tried to improve the existent $2 \mathrm{~b}-\mathrm{RAD}$ library approach. At the present times, multiplex sequencing methods have been developed for NGS using either barcoded adapters, especially in reduced representation library [5-13], or indexed amplification primers [16-19]. We attempted to combine these two methods together to develop a new multiplexing $2 \mathrm{~b}-\mathrm{RAD}$ library approach. Twelve adapters with different barcodes were designed in this research and 12 samples could be pooled together. To validate the new approach, we prepared 24 2b-RAD libraries containing 285 samples from a rice (Oryza sativa .L) F2 population. The population was genotyped and a genetic linkage map was constructed. The markers were aligned with Nipponbare reference to measure the accuracy of the genetics map. Moreover, series of bioinformatics simulate analysis of $2 \mathrm{~b}-\mathrm{RAD}$ tags based on ten plants and ten animals' reference genomes were carried out. Processing with methylation-sensitive REs, it is difficult to predict which recognition sites would be digested in GBS. So we only compared RAD and 2b-RAD tags on these species. The tag numbers and distributions were analyzed specifically.

\section{Methods}

\section{Comparison of RAD and $2 b-R A D$ tags}

The REs recognition sites, including EcoRI, Sbfl and HindIII for RAD, BsaXI and Alfl for 2b-RAD, were detected on the reference genomes containing 10 plants and 10 animals. Each sequence of "GAATTC" (EcoRI), "CCTGCAGG" (Sbfl) and "AAGCTT" (HindIII) tested on the genomes was regarded as one recognition site, producing two tags for RAD [5]; each sequence of "ACNNNNNCTCC", "GGAGNNNNNGT" (both for BsaXI) and "GCANNNNNNTGC" (Alfl) was regarded as one recognition site, producing one tag for $2 \mathrm{~b}-\mathrm{RAD}$, respectively.

The EcoRI was one of the most commonly used REs for RAD. For 2b-RAD, the recognition sites of BsaXI were much more than Alfl by simulation. The recognition bases of EcoRI and BsaXI were both six. So the application potential of EcoRI and BsaXI were compared. The genomes size was divided by EcoRI sites numbers. The average fragment length was approximately $4000 \mathrm{bp}$ of the 20 species, indicating that EcoRI recognition sites located once per 4000 bp. Each 4000 bp sequence was regarded as a window and represented a simulated tiny scaffold. The recognition sites equaled 0,1 and more than 1 in these scaffold denoted different potentiality of assembly. Zero indicated that the scaffold was impossible to be connected to any chromosome; one indicated the scaffold could be connected with no orientation; more than one indicated potential perfect assembly with an orientation. The sites of EcoRI and 
BsaXI were detected in these tiny scaffolds to estimate their potentiality for chromosome assembly.

\section{F2 populations}

The F2 population materials including two F0 parents and 277 F2 progeny were gained from the rice genetic breeding laboratory of China Agricultural University. Of the two parents, the female was Nipponbare while the male was a stable recombinant inbred line for five generations of Oryza sativa spp. Japonica line whose ancestry derived from a cross between two Japonica varieties. One was Nipponbare, and the other was a Chinese landrace with the name of Mayi danru. The parents and their progeny, altogether 279 DNA were extracted from their fresh leaves according to Doyle's protocol [20]. The DNA only with a lowest concentration of $50 \mathrm{ng} / \mu \mathrm{L}$ and no degradation were able to be applied for the following 2b-RAD library.

\section{Adapter design}

To create a simple and quick $2 \mathrm{~b}-\mathrm{RAD}$ library approach with pooling procedure, we designed two kinds of adapters. Adapter1 with 5-9 bp barcodes was complementary to the Illumina multiplexing PCR primer 1.0; adapter2 was complementary to index primer. The digested fragment of BsaXI was 33 bp with 3 bp random overhangs on the 3 ' ends. The sequences of the adapter1 were:

\section{5'-ACACTCTTTCCCTACACGACGCTCTTCCGAT CTxxxxxNNN-3' and 5'-yyyyyAGATCGGAAGAGC GTCGTGTAGGGAAAGAGTGT-3", the "xxxxx" and "yyyyy" denoted the barcode and its complement sequences. The NNN was complementary to the $3 \mathrm{bp}$ sticky end generated by BsaXI ("N" was a random base of A, G, C, T and there were 64 kinds of combinations altogether). The adapter2 was: \\ 5' - AGATCGGAAGAGCACACGTCTGAACTCCAG TCAC $-3^{\prime}$ and $5^{\prime}$-GTGACTGGAGTTCAGACGTG TGCTCTTCCGATCTNNN-3'. The annealed adapter1 and adapter 2 were adjusted to $5 \mu \mathrm{M}$ as working concentration.}

The BsaXI digested fragment was only 33 bp and it would be completely sequenced by single-end $50 \mathrm{bp}$ (SE 50 ), so a little longer ( $9 \mathrm{bp}$ ) barcodes would not reduce the tag sequence. The barcodes were designed according to Poland JA's criteria [12] and made slight modification. (1) The lengths of barcodes were different form 5 to 9 bp to maximize the balance of bases at each position, especially in the BsaXI recognition sites. (2) The barcodes must be two or more bp different from all other barcodes. (3) The barcodes can't contain or recreate (after ligation step) BsaXI restriction site. A set of 12 barcode sequence were designed (Additional file 1).

\section{2b-RAD library preparation}

The concentrations of all DNA samples were adjusted to $50 \mathrm{ng} / \mu \mathrm{L}$. The DNA (200 ng) was digested in $10 \mu \mathrm{L}$ reaction volume of NEB Buffer 4 with $1 \mathrm{U}$ BsaXI (New England BioLabs Inc, Catalog \# R0609L) at $37^{\circ} \mathrm{C}$ for $2 \mathrm{~h}$. An additional DNA was digested simultaneously to detect the digestion efficiency by $1 \%$ agarose gel electrophoresis. The primary DNA band disappeared and became disperse, indicating a successful digestion. Then the ligation reaction was completed in the same tube as the digestion, combining the remaining digested DNA with $2 \mu \mathrm{L}$ of the adapter1, $2 \mu \mathrm{L}$ of the adapter2, $2 \mu \mathrm{L}$ ligase buffer and $400 \mathrm{U}$ of the T4 ligase (New England BioLabs Inc, Catalog \# M0202L). BsaxI could not be inactivated by heating, so the digested productions was recommended performing the ligations in $4^{\circ} \mathrm{C}$ for 1 hour, then hold on ice [14].

For exploring the relationship between sequencing data size and enzyme sites coverage, each parents repeated 4 times in library experiment. All 285 DNA samples (277 progeny +4 female +4 male) were divided into 24 groups. Each group contained 12 samples and the last group contained 9 samples. The samples in each group were ligated with different 12 adapters.

Twelve samples in a group were pooled together when they were purified using purification kit (QIAquick PCR Purification Kit, Catalog \# 28106). The ligated productions were completely combined to keep the amount of pooled DNA as $1 \sim 1.5 \mu \mathrm{g}$, which was sufficient for the following PCR procedure. The samples in each group with different barcode adapters were gathered to a tube which had been added the wash buffer of purification kit beforehand. Then the pooled DNA was purified according to the manufacturer's instructions and eluted in $25 \mu \mathrm{L}$ EB (elution buffer).

The Illumina multiplexing PCR primers were used for amplification. The sequences were:

\section{5'-AATGATACGGCGACCACCGAGATCTACAC TCTTTCCCTACACGACGCTCTTCCGATCT-3' (multiplexing PCR primer 1.0) and 5'-CAAGCAGAAGACGGCATACGAGATXX XXXXGTGACTGGAGTTCAGACGTGTGCTC TTCCGATCT-3' (index primer), where the six " $X$ " represented a $6 \mathrm{bp}$ index. The index primer sequences were derived from a BGI patent (http://www.google.ca/ patents/WO2012037880A1?hl=zh-CN\&cl=en) [21]. For each PCR, we combined 50 ng of pooled DNA, $1 \mu \mathrm{L}$ of each primer $(10 \mu \mathrm{M})$, buffers, nuclease-free water and Phusion polymerase (New England BioLabs Inc,}


M0531) at a final $50 \mu \mathrm{L}$ total volume. Temperature cycling consisted of $98^{\circ} \mathrm{C}$ for 30 seconds followed by 12 cycles of $98^{\circ} \mathrm{C}$ for 30 seconds, $65^{\circ} \mathrm{C}$ for 30 seconds, $72^{\circ} \mathrm{C}$ for 30 seconds with a final Taq extension step at $72^{\circ} \mathrm{C}$ for 5 minutes. Four kinds of index primers were used in this study for distinguishing the libraries (Additional file 1). The expected tag fragment after PCR was from $160 \mathrm{bp}$ to $164 \mathrm{bp}$ (5-9 bp barcode). PCR production was detecting by $2 \%$ agarose gel electrophoresis, and then the 150-200 bp bands were cut and purified by the purification gel kit (QIAquick Gel Extraction Kit, 28704) and eluted in $30 \mu \mathrm{L} \mathrm{EB}$.

The quantify molarity and library fragment size distribution of cleaned DNA were detected by an Agilent Bioanalyzer. Quantification was conducted by qPCR. SE50 sequencing of four 12-plex libraries per flowcell channel lane was performed on Hiseq 2000 platform (Illumina, Inc.). Totally six lanes were used.

The detailed protocol of whole 2b-RAD library procedure was available on Additional file 2. The complementary relationships of all the sequence used in 2b-RAD library were showed on Figure 1.

\section{Filtering raw sequence data}

The reads filtering steps were performed by our own Perl scrip as follows. (1) Matched one of the 12 barcodes allowing one mismatch. After the reads were assigned into each sample, the 5-9 bases barcode were removed. (2) The reads following on the heels of the barcode should perfectly matched BsaXI fragment "NNNNN NNNNNNNACNNNNNCTCCNNNNNNNNNN" or "NNNNNNNNNNGGAGNNNNNGTNNNNNNNNN NNN" (these "N" means random sequences of BsaXI digestion fragments) with no "Ns" ("Ns" means the bases which were failed to be sequenced by Hiseq 2000). Then the reminder bases of each read were deleted. Two kinds of $33 \mathrm{bp}$ tags containing the recognition sites "ACNNNNNCTCC" or "GGAGNNNNNGT" were obtained. They were regarded as high quality reads.

The two cohesive ends of BsaXI digestion fragment were identical, so the adapter1 was probably ligated to any ends of the fragment, as well as the adapter2. So a fragment with many copies after DNA extracting and digestion, was ligated to adapters of couple of possibilities. However, only the fragment which was ligated adapter1 and adapter 2 simultaneously could be PCR amplified. The fragment with only 33 bp length, was probably sequenced both form plus strand and minus strand (Figure 2). In order to solve this problem, all the tags contained "GGAGNNNNNGT" were translated to their reverse compliment sequences, namely, the form of "ACNNNNNCTCC". And then the tags contained only one strand of the digestion fragments.

\section{The tag mapping}

For estimating BsaXI sites coverage, firstly, simulant detections of the BsaXI sites in Nipponbare reference genome were carried out. The $33 \mathrm{bp}$ fragments in reference containing recognition sites of "GGAGNNNNNGT" or "ACNNNNNCTCC" were picked out. Then these sequences were mapped to reference using Bowtie (version 0.12.7) with the parameter of -m 1 -v 2 allowing 2 mismatches. The sequences which aligned with only one location were regarded as unique tags and the expected potential markers.

Secondly, the high quality reads of 285 samples were mapped to Nipponbare reference (Bowtie $-\mathrm{m} 1-\mathrm{v} 1$ ). The mapped reads were detected whether they covered simulant tags.

To look for the relationship between sequencing data size and BsaXI sites coverage, a testing was performed based on several combinations of 4 repeated female samples. The female was Nipponbare, as well as the reference genome. Therefore the testing could accurately reflect the relationship.

After digestion, the fragments between two recognition sites also possessed sticky end "NNN" as follows.

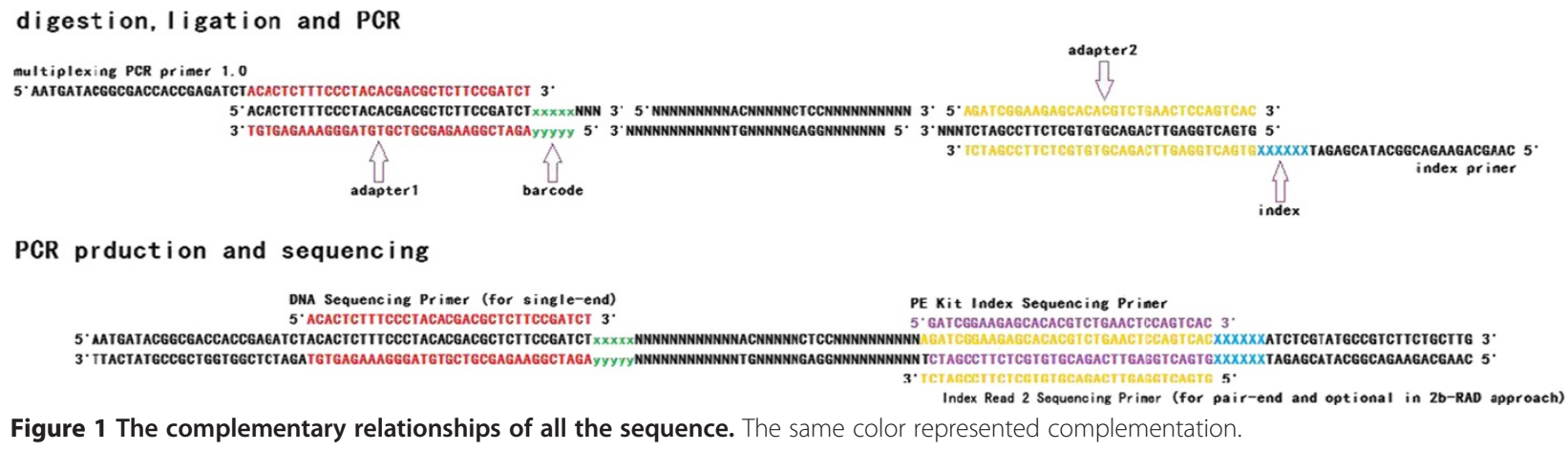

Figure 1 The complementary relationships of all the sequence. The same color represented complementation. 


\section{----REs----NNN NNNNNNNNNNNNNNNNNNN ----REs----NNN NNN----REs---- NNNNNNNNNNNNNNNNNNN NNN----REs----}

The structure made the fragments possible to be ligated with adapters and performed PCR amplification. If their length was nearly $33 \mathrm{bp}$, they would not be removed in the agarose gel size selection and be sequenced. So the reads matched barcodes while not matched BsaXI recognition sites were analyzed. After removing redundant sequences (barcode and part of atapter2), they were mapped to Nipponbare reference genome (Bowtie - m 1 -v 1).

\section{SNP calling with and without reference}

Any two of the female samples combination could cover nearly $100 \%$ of the simulant REs sites, so we combined female- 2 and female- 3 together (with the lowest data size) to perform SNP-calling. Same treatment to male samples of male- 2 and male- 4 were performed.

The SNP calling without references were performed using Stacks (version 1.01) [22] with four programs. The parameters were -m $2-\mathrm{M} 2-\mathrm{N} 1$ for ustacks, $-\mathrm{n} 2$ for cstacks, default for sstacks and -m 2 -t F2 -o joinmap -c -min_hom_seqs 2 -min_het_seqs $0.010-$ max_het_seqs 0.011 for genotypes. The "-m" in genotypes means minimum reads depth for genotype. We used $-\mathrm{m} 2$ as the depth and the automated corrections system $(-\mathrm{c})$ in consideration of the lower sequencing depth of the progeny. The markers of aa $\mathrm{x}$ bb style were selected for genetic map. Meanwhile, the female tags of these markers were mapped to Nipponbare (Bowtie -m 1 -v 1).

To construct the genetic linkage map, the markers derived from Stacks were pretreated. The markers with less than $20 \%$ missing rate $(<54$ samples) were preserved. The linkage analysis was performed using JoinMap (version 4.1, http://www.Joinmap.nl). The SNP genotypes in F2 population were expected to segregate at a $1: 2: 1$ ratio. Distorted markers $(\mathrm{P}<0.01)$ were filtered by $\mathrm{X}^{2}$ test. The parameters using JoinMap were "independence LOD" for grouping method, "regression mapping" for mapping algorithm, "Kosambi's" for mapping function method. The groups with less than 5 markers were discarded. Each linkage map marker's location in alignment result was retrieved to measure accuracy of the genetic map. Of the sorted markers via genetic distances in each group, the one which was significantly opposite the mainstream of the order (more than $2 \mathrm{Mbp}$ to its adjacent two markers) was regarded as "order error" marker.

To roughly evaluate SNP numbers between two parents, the other way for genotyping relied on reference genome. Firstly, reads of two parents were mapped to the Nipponbare reference using SOAP2 (version 2.21) with default. The aligned reads were performed SNP calling using SOAPsnp (version 1.03) with default. The SNPs in cns file (SOAPsnp result file) were preserved only when

\section{a. digested DNA}
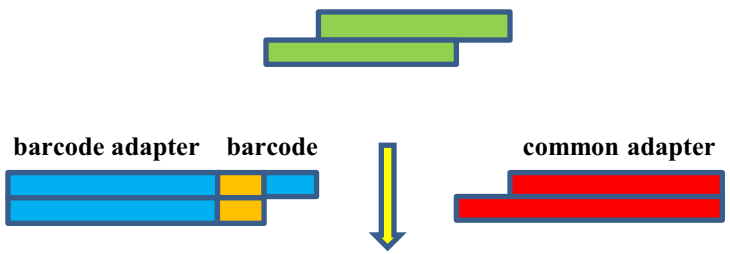

ligated production
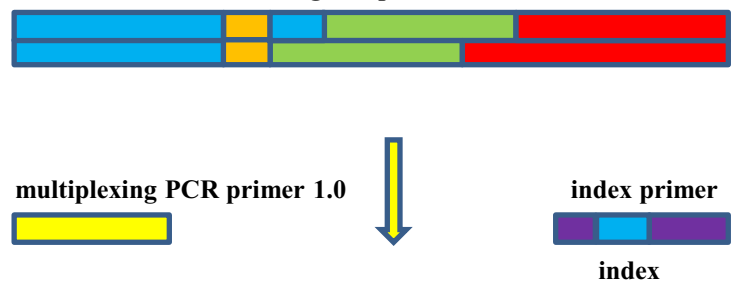

PCR production

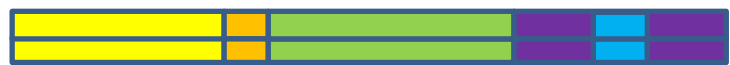

\section{b. digested DNA}
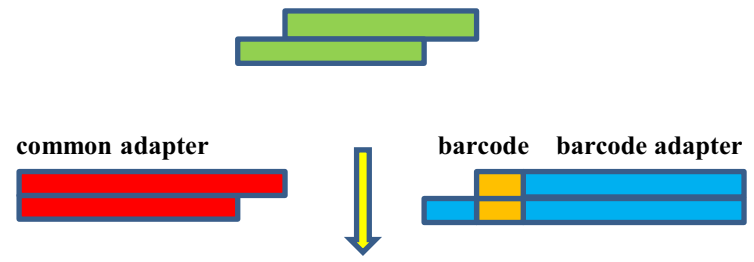

ligated production
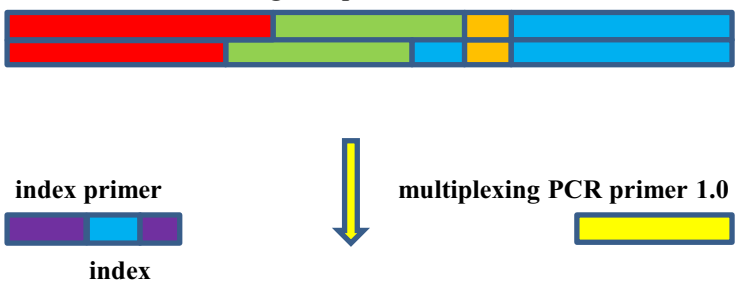

PCR production

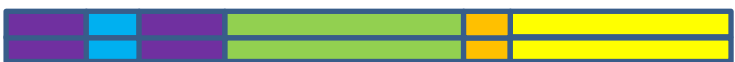

Figure $\mathbf{2}$ The tag with many copies which was probably sequenced both form plus strand and minus strand. The $\mathbf{a}$ and $\mathbf{b}$ revealed that the adapter 1 and adapter 2 may ligate to any ends of the 33 bp digested fragment and the tag would be sequenced from any $5^{\prime}$ end. 
the quality score of consensus genotype were more than 10. After integrating their SNP results, the genotype of aa $\mathrm{x}$ bb style between two parents were obtained. The two results between SOAPsnp and Stacks were compared.

\section{Result}

\section{Comparison of RAD and $2 b-R A D$ tags}

The REs recognition sites of RAD and 2b-RAD in simulated species were listed in Table 1 . The tag of RAD was twice the number of its recognition sites. On average, the BsaXI had the most recognition sites numbers among the five REs. However, the tag numbers of EcoRI and HindIII were more than BsaXI. The recognition sites of Alfl were much less than BsaXI, making it no significance applications in high density linkage map, so we only used BsaXI in the following simulation and experiment.

The average value that genome size divided by EcoRI recognition sites number was $3647 \mathrm{bp}$ in plants and 3833 bp in animals. We employed 4000 bp as a window to detect the presence numbers of EcoRI and BsaXI recognition sites. Overall, 4749893 windows were detected. The windows where EcoRI recognition sites numbers equaled 0,1 and more than 1 were 1839924 (39\%), 1566583 (33\%) and $1343386(28 \%)$. The equivalents for BsaXI were 1198293 (25\%), 1340862 (28\%) and 2210738 (47\%).

In addition, we detected the recognition sites numbers of $B s a \mathrm{XI}$ in the windows where EcoRI recognition sites numbers were 0 or 1 . Zero or one meant that these windows were impossible to be perfectly assembled to a chromosome. There were 825203 (EcoRI $=0$ and $B s a \mathrm{XI}>1)$ and 1218063 windows (EcoRI $=1$ and $B s a \mathrm{XI}>0)$, and the percentages were $45 \%(825203 / 1839924)$ and $78 \%$ (1218063/1566583). The simulations of EcoRI $=0, B s a X I>1$ and $E c o$ RI $=1, B s a \mathrm{XI}>0$ both indicated that the windows contained more than one REs recognition sites. This fact revealed that the perfect assembled windows were significantly increased after adding the BsaXI tags. The windows contained at least two recognition sites was up to $71.3 \%$ of the total windows.

Table 1 The REs recognition sites and tag numbers of RAD and 2b-RAD in simulated species

\begin{tabular}{|c|c|c|c|c|c|c|c|}
\hline Species & Size (G) & EcoRI & $S b f l$ & HindIII & BsaXI & Alfl & Version \\
\hline Arabidopsis thaliana & 0.12 & 37057 & 627 & 67057 & 39826 & 12601 & TAIR9 \\
\hline Cucumis sativus & 0.24 & 69944 & 1197 & 94344 & 65249 & 23762 & v2.3 \\
\hline Theobroma cacao & 0.33 & 90253 & 1572 & 173770 & 81810 & 39800 & CIRAD_V0.9 \\
\hline Oryza sativa & 0.37 & 88471 & 5541 & 110084 & 180848 & 83465 & IRGSP-7.0 \\
\hline Vitis vinifera & 0.49 & 143785 & 2594 & 228694 & 155893 & 62070 & Genoscope \\
\hline Cajanus cajan & 0.61 & 161249 & 1652 & 232261 & 162320 & 62119 & v1.0 \\
\hline Solanum tuberosum & 0.73 & 233925 & 2957 & 273063 & 212095 & 70042 & v3.4 \\
\hline Sorghum bicolor & 0.74 & 200543 & 11693 & 308083 & 379985 & 151244 & phytozome_v7.0 \\
\hline Glycine max & 0.97 & 309451 & 3844 & 422507 & 289940 & 140204 & phytozome_v7.0 \\
\hline Zea mays & 2.06 & 490315 & 50773 & 768456 & 1151014 & 444673 & AGl_5a \\
\hline Total & 6.65 & 1824993 & 82450 & 2678319 & 2718980 & 1089980 & \\
\hline Average & 0.67 & 182499 & 8245 & 267831 & 271898 & 108998 & \\
\hline Average tag number & & 364998 & 16490 & 535663 & 271898 & 108998 & \\
\hline Drosophila melanogaster & 0.17 & 44863 & 2948 & 45913 & 58189 & 50403 & RGSC3.4 \\
\hline Apis mellifera & 0.23 & 97174 & 551 & 49977 & 58053 & 21237 & AnoCar2.0 \\
\hline Anopheles gambiae & 0.27 & 53807 & 3164 & 87297 & 100574 & 80148 & Galgal4 \\
\hline Takifugu rubripes & 0.39 & 75238 & 20718 & 95182 & 210480 & 103943 & FUGU4 \\
\hline Bombyx mori & 0.48 & 112113 & 2041 & 121288 & 96793 & 47329 & AgamP3 \\
\hline Gallus gallus & 1.05 & 277001 & 48010 & 403125 & 453890 & 400359 & NCBIM37 \\
\hline Danio rerio & 1.41 & 255801 & 30677 & 427420 & 426194 & 339437 & v1.0 \\
\hline Mus musculus & 2.72 & 758802 & 60302 & 842075 & 1331056 & 607106 & 2.0 \\
\hline Rattus norvegicus & 2.72 & 817664 & 58283 & 818955 & 1257212 & 595706 & r5.27 \\
\hline Homo sapiens & 3.10 & 778227 & 77095 & 837133 & 1292381 & 583536 & $\mathrm{~V} 2.0$ \\
\hline Total & 12.54 & 3270690 & 303789 & 3728365 & 5284822 & 2829204 & \\
\hline Average & 1.17 & 327069 & 30378 & 372836 & 528482 & 282920 & \\
\hline Average tag number & & 654138 & 60758 & 745673 & 528482 & 282920 & \\
\hline
\end{tabular}


The detailed windows simulation was showed on Figure 3 and Additional file 3.

\section{Experimental $2 b-R A D$ results}

Overall, the 24 libraries of 6 lanes produced 860368972 raw reads, equaling to $43 \mathrm{Gbp}$ raw data. Each lane produced 7.2 Gbp data on average. The number of the reads matched barcode was 813497032 (94.6\%). The reads matched both barcode and BsaXI recognition sites were 570094697 (66.3\%). These reads were regarded as high quality reads, including 305405977 (53.6\%) reads which were the style of "ACNNNNNCTCC" and 264688720 $(46.4 \%)$ reads which were "GGAGNNNNNGT". The 264688720 reads were translated to the style of “ACNNNNNCTCC" before genotyping. Totally 290274275 (33.7\%) raw reads were filtered. Of these filtered reads, 46817940 (16.1\%) were filtered on account of barcode match failure and 243402335 (83.9\%) were filtered by BsaXI sites match failure.

Each sample's reads divided by barcode were from 1815973 to 5303555 . The ratio of the maximum divided by minimum was 2.9. After BsaXI sites matching step, the high quality reads were from 862169 to 4195194, and the ratio was 4.9 (Figure 4). On average, each sample was given 2000332 high quality reads, equaling to $66 \mathrm{Mbp}$.

The detailed reads data of 285 samples and 24 libraries were listed in Additional file 4.

\section{Comparison of observed and expected 2b-RAD tag}

The total expected BsaXI sites in Nipponbare reference were 180848, including 105142 unique sites. All together 570,094,697 high quality reads of 285 samples were mapped to Nipponbare reference. The 550108260 (96.5\%) reads could be aligned with the Nipponbare reference. On average, these reads covered $82.5 \%$ (149270) of the total expected BsaXI recognition sites and $84.2 \%$ (88503) of the unique sites (Additional file 5). The actual average depth of each BsaXI site was 10.67 (550108260/285/180848).

The combinations of 4 repeated female samples' results were showed in Table 2.

The 243402335 filtered reads by BsaXI sites matching step were also mapped to reference. The result revealed that $209287752(86.0 \%)$ of these reads could be aligned with reference. Namely, $72.1 \%$ of the total filtered reads were able to be mapped to reference but failed to match BsaXI recognition sites. This fact may indicate that these reads were the fragments between two $B s a \mathrm{XI}$ recognition sites.

\section{Genotyping and genetic mapping}

In the cstacks procedure, 118289 catalogs were produced by the two parents' reads. After genotypes procedure, the markers of aa xx bb style were 3598 and all of them could be successfully aligned with reference including 3580

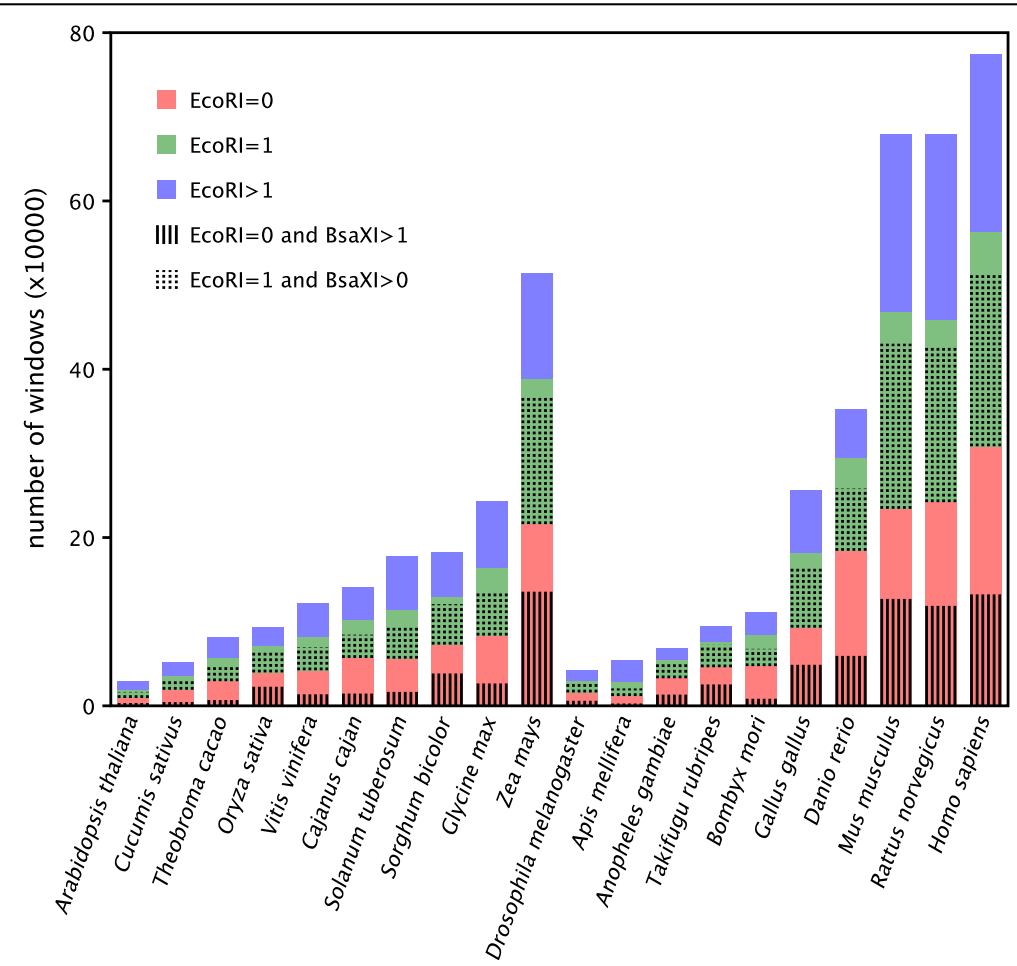

Figure 3 The assemble potentiality simulation of EcoRI and BsaXI in $\mathbf{2 0}$ species. The different color represents the different windows where EcoRI recognition sites were 0,1 or more than 1 . The small figure of vertical lines represented the windows where EcoRI recognition sites were 0 and BsaXI were more than 1. The small figure of spots represented the windows where EcoRI recognition sites were 1 and $B$ saXI were more than 0. 


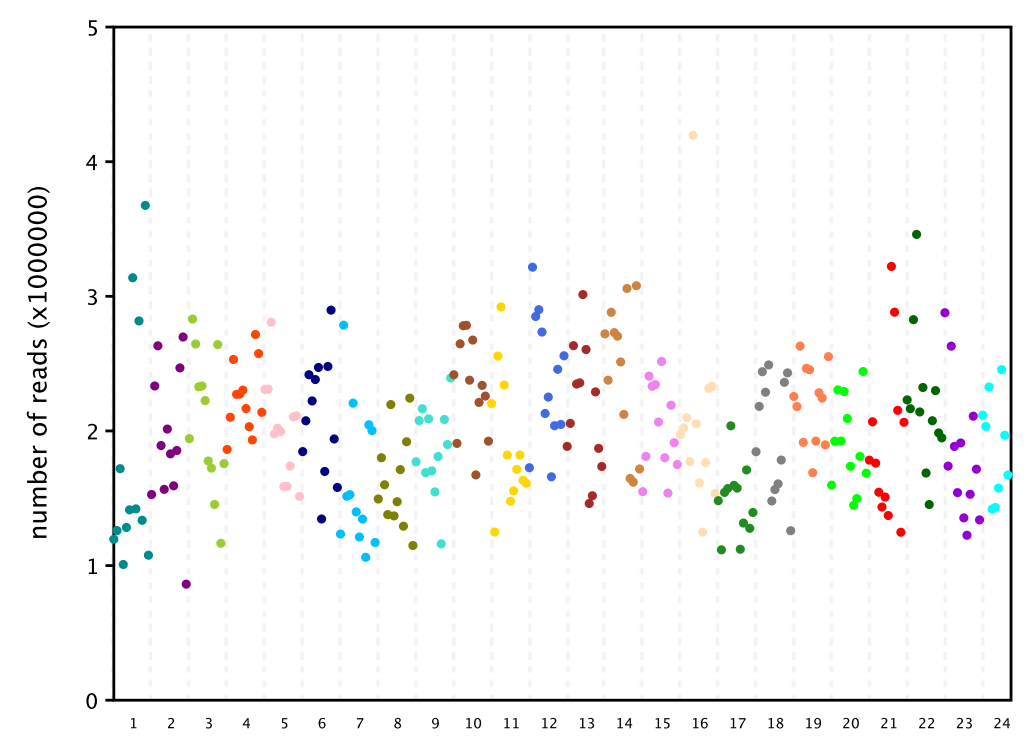

Figure 4 The high quality reads numbers of the $\mathbf{2 8 5}$ samples. The different colors represent different libraries.

unique markers. Eighteen markers were mapped to more than one location. For the 3580 unique markers, there were 239 markers each contained two SNPs. The alignment result revealed another fact that 33 pairs of markers had overlaps. These overlaps were attributed to two close BsaXI recognition sites in a chromosome (Figure 5). The probability of the overlapped makers was $0.9 \%$ (33/3598). Totally 3804 SNPs were confirmed. The SNPs yielded by SOAPsnp were 4769. There were 2633 SNPs loci were both in SOAPsnp result and Stacks results. All of the markers information was showed in Additional file 6.

For the 3598 markers, the total missing rate was $18.9 \%$ (total missing alleles divided by $3598 \times 277$ ). The markers with less than $20 \%$ missing rate $(<54$ samples) were 2547. By $\chi^{2}$ test, 1391 markers survived. Six markers were assigned to some tiny groups (markers were less than 5).
The reminder 1385 markers were divided into 15 groups and the corresponding LOD value ranged from 4 to 31 . Ten groups were divided by a lower LOD value of 4 or 5 ; one group was divided by 9; and another four groups were divided by 25,30 or 31 . One marker was aligned with inappropriate chromosome (marker3869) and 112 markers (8\%) revealed significant order errors. Totally there were 1384 markers which were able to be aligned with correct chromosome (Figure 6). The detailed information of 1385 markers was available on Additional file 7.

\section{Discussion}

\section{2b-RAD library approach improvement}

The 2b-RAD approach was invented by Wang in 2012 and was proven as a simple and flexible method for genome-wide genotyping [14]. However, this technique was rarely used in the published researches. Lacking of

\section{Table 2 The combinations of 4 repeated female}

\begin{tabular}{|c|c|c|c|c|}
\hline Reads number & Depth & Combination & Covered sites & Coverage rate \\
\hline 2333752 & 12.8 & fe-1 & 151387 & $83.71 \%$ \\
\hline 2225621 & 12.2 & $\mathrm{fe}-2$ & 151352 & $83.69 \%$ \\
\hline 1575530 & 8.5 & $\mathrm{fe}-3$ & 150720 & $83.34 \%$ \\
\hline 2456057 & 13.4 & fe-4 & 156650 & $86.62 \%$ \\
\hline 3909282 & 21.6 & $\mathrm{fe}-3+\mathrm{fe}-1$ & 178654 & $98.79 \%$ \\
\hline 3801151 & 21.0 & $f e-3+f e-2$ & 177239 & $98.00 \%$ \\
\hline 4031587 & 22.3 & $f e-3+f e-4$ & 178946 & $98.95 \%$ \\
\hline 6134903 & 33.9 & $f e-3+f e-1+f e-2$ & 180098 & $99.59 \%$ \\
\hline 8590960 & 47.5 & $\mathrm{fe}-3+\mathrm{fe}-1+\mathrm{fe}-2+\mathrm{fe}-4$ & 180104 & $99.59 \%$ \\
\hline
\end{tabular}

"fe" means female parent; "reads number" means the reads which were able to be mapped to reference; "depth" equals to reads number divided by the total expected BsaXI recognition sites; "covered sites" means the BsaXI sites in reference which was covered by high quality reads; "coverage rate" equals to the "covered sites" divided by the total expected BsaXI recognition sites. 


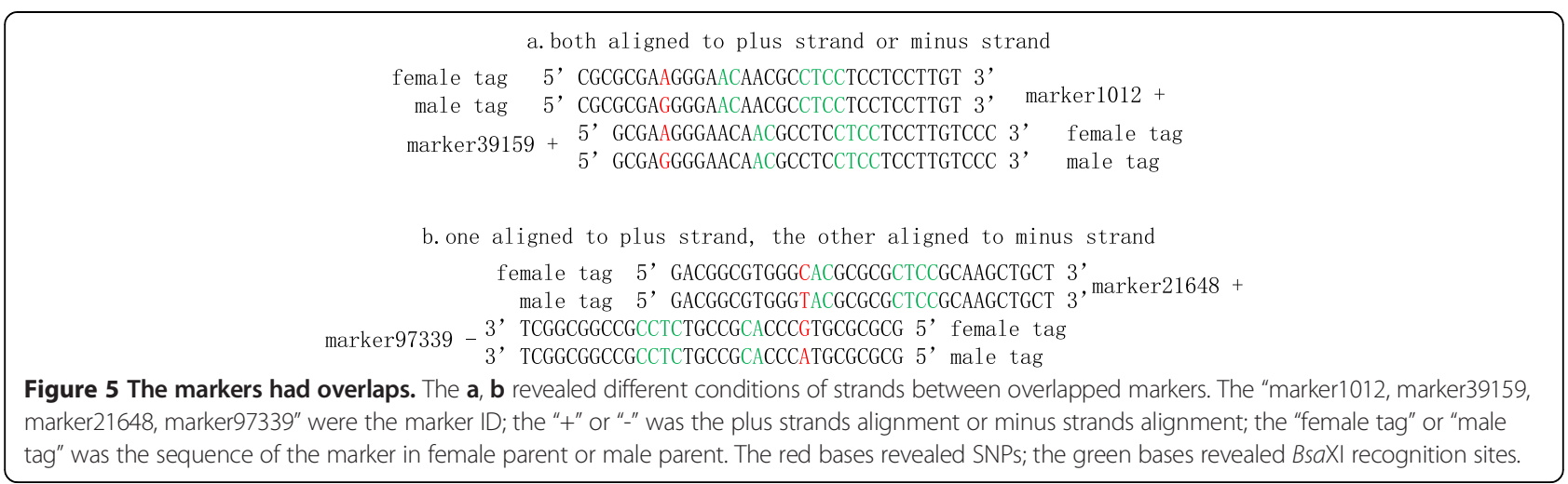

pooling library approach as other reduced representation libraries, made it take more manpower and time, and may partly explain the reason.

The existent reduced representation libraries, such as RAD, GBS, two enzyme GBS and double digested RAD, were all pooling library sequencing techniques. The barcode adapters of RAD and double digested RAD were phosphorylated in $5^{\prime}$ end, which made them more expensive in adapter cost than GBS and two enzyme GBS whose adapters were not phosphorylated. Moreover, to mix many libraries for multiplexing sequencing in a flowcell lane, the indexed primer was widely used in

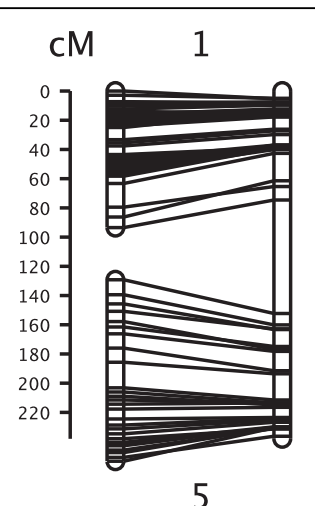

5

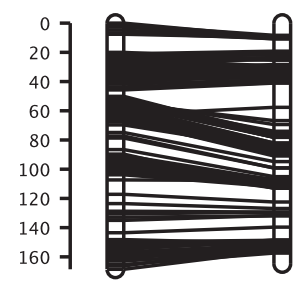

9

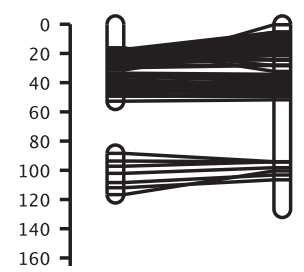

3

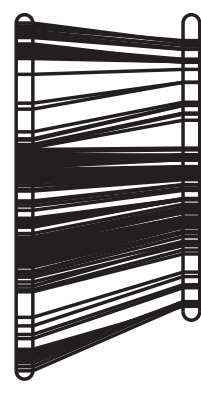

6

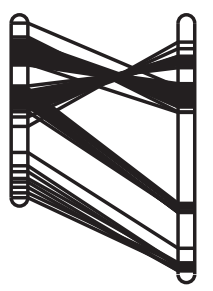

10

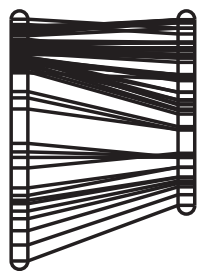

11

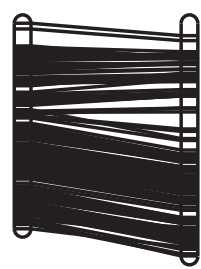

12

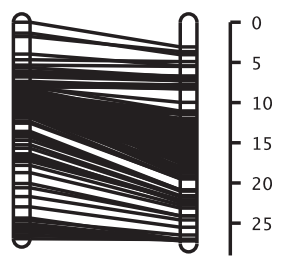

Figure 6 The 1384 markers of 15 groups which were able to be aligned with Nipponbare reference. The left vertical line was the linkage group and the right was the reference chromosome. 
Illumina Hiseq platform. For saving library cost, we used the non-phosphorylated adapters and the index primer. The combination of these two methods achieved that using 12 barcode adapters completed the pooling library and multiplexing sequencing rather than using 96 or 48 barcode adapters. However, this approach was not only the method for improving the existent $2 \mathrm{~b}-\mathrm{RAD}$ library approach. The barcode adapters and PCR primers like the style of RAD, GBS, two enzyme GBS or double digested RAD methods could also be modified to fit for 2b-RAD. The method in this research was just a comparatively cost-effective method for materials costs.

For library experiment, the method comprised digestion, ligation, pooling, PCR and size selection. Comparing with Wang's approach, the method added a size selections procedure, but reduced a step of PCR. The size selection was inevitably, because the fragments between two REs recognition sites could also be ligated with adapter and amplified. They had a large amount reveled by the agarose gel electrophoresis outside 150-200 bp (Additional file 2 Figure 2).

Anyhow, the improved library approach reduced manpower and time cost. Completing a library including 12 samples took only 5 hours. Achieving 8 libraries containing 96 samples within a day wouldn't be a problem. So this method was suit for large sample size population.

\section{The merits and demerits of the method}

By filtered step, $33.74 \%$ of the raw reads were filtered. The $72.1 \%$ of the filtered reads were mapped to reference, indicating that most of these reads may be the fragments between two BsaXI recognition sites. For easy operation, 150-200 bp DNA bands were selected in library procedure. The bands in this region which were less than 160 or more than 164 were probably the fragments between two BsaXI recognition sites and so useless data increased. Using more accurate DNA markers to select the 160164 bp fragments, could reduce the useless data.

The evenness of each sample reads was important. The reads number differing too big could make analysis difficult and inaccurate. Of the high quality reads, the ratio about the maximum reads numbers divided by minimum reads number was 4.9 , showing a high evenness. The sample in each library was only 12, making it easy to keeping the same amount mixing. A library containing 96 samples were not so easy to keep evenness in experimental operation. This was another reason why we used only 12 barcodes for a library. Of course, the barcode adapters could be increased as needed.

The average depth of each BsaXI site was 10.67. For 3598 Stacks markers, 1051 markers (29.2\%) were filtered with a higher missing ratio and 1156 markers (32.1\%) were filtered by $x^{2}$ test. For the genetic map result, four groups were divided by a higher LOD value. Some genetics group could not directly gather into a chromosome (chromosome 1, 4 and 9). The genetic group corresponding to chromosome 6 revealed that many markers showed order error. For chromosome 9, fewer markers were assigned to one chromosome arm. Meanwhile, the alignment result of all Stacks markers revealed wide distribution in chromosome (Additional file 6). These demerits indicated that the progeny's genotypes were not accurate enough. It may attribute to the lower sequencing depth. By the detection of REs sites, we found BsaXI sites were more than EcoRI, especially in Oryza sativa. The average depth of $10.67 \times$ was a bit lower to confirm heterozygote genotypes. The low-quality data of progeny made that 61.3\% of the Stacks marker were filtered and just right most of the markers in one arm of chromosome 9 were filtered. To overcome the shortcomings, the sequencing data should be increased. However, only one marker was aligned to inappropriate chromosome and 112 markers revealed significant order errors, proving the practicability of the approach.

The SNPs between two parents calculated by SOAPsnp were 4769, while by Stacks were 3598. The SOAPsnp was not the professional software for reduced representation genome analysis. It was used for roughly estimating the total SNPs in this research. The comparing results between SOAPsnp and Stacks revealed that the actual SNPs were approximately 3000 to 5000 . The markers for genetic map were 1391. Of the published RAD or GBS researches for genetic map analysis, the final marker numbers were from several hundreds to thousands [10,23-29]. The female parent was Nipponbare, while the male could trace its ancestry to Nipponbare. The two parents had close relationships. However, 3598 markers were obtained which were comparable to published RAD or GBS researches. Using more distantly related parents would receive more SNPs.

The alignment result of Stacks markers revealed that 33 pairs of markers had overlaps. They could be assembled to long tags to discover SNPs. But the probability of the overlapped makers was too low for more valuable applications.

\section{The practical application in the future}

The most important function of reduced representation libraries is genotyping, namely, the marker (main SNPs) discovery. Based on these markers, the genetic map can be constructed, comprising two main applications: QTL fine mapping [23-27] and de novo chromosomes assembly [28,29].

One recognition sites of RAD could produce two tags, making HindIII had the most simulant tags in 20 species on average. Meanwhile, the RAD tags were long enough to carry out single-end $90 \mathrm{bp}$ sequencing. In addition, RAD had another application - paired end sequencing for 
de novo assembly and marker design [30,31]. Possessing more and long tags means more chance to obtain SNPs, so RAD was more effective than 2b-RAD in SNP discovery. However, the two tags produced by one RAD REs site located together. Two tags which stand together amount to one marker in linkage map. So marker density for genetic map is mainly depending on the numbers of scattered recognition sites rather than tags. The REs for RAD all possess palindromic recognition sites, as well as Alfl, while BsaXI doesn't. Two kinds of recognition sites result that $B s a \mathrm{XI}$ have the most recognition sites, making $2 \mathrm{~b}-\mathrm{RAD}$ approach is potentially more effectively than RAD for constructing high density genetic map. It should be noted that the progeny's genotypes were not accurate in this research and may be attributed to the lower sequencing depth. The combinations of 4 repeated female samples' results revealed that doubled data size increased the BsaXI sites coverage to almost 100\%. To solve the problem for gaining more accurate data, the one way is to increase sequencing data by doubling lanes. The samples can be reduced to 24 of 2 libraries in a single lane and the total lanes rise to 12 . In addition, the design of adapters and PCR primers in this research made $2 \mathrm{~b}-\mathrm{RAD}$ tags could be either performed single-end sequencing or paired-end sequencing (Figure 1). The PE1 and PE2 reads from paired-end $50 \mathrm{bp}$ (PE50) sequencing would be entirely over merged. Hence, except adding flowcell lanes, the other way to double data size is to use paired-end sequencing. In commonly, with a same total data size, a PE lane is more inexpensive than two SE lanes. The paired-end sequencing strategy for $2 b-R A D$ with a significant cost advantage is worth popularizing in the future applications.

The marker density of genetic map is important for de novo chromosomes assembly because possessing more markers for a contig or scaffold means more chance to be perfectly assembled. By the assembly simulation, the tiny scaffolds which were potentially perfectly assembled were $28 \%$ for $E c o$ RI and $47 \%$ for BsaXI, indicating 2b-RAD using $B s a \mathrm{XI}$ were more effective in chromosomes assembly. Of course, simultaneous use of EcoRI and BsaXI markers, the perfect assembly rate rose to $71.3 \%$.

Using several hundreds of DNA markers is common and sufficient for typical QTL mapping studies. Therefore, RAD-QTL approach often use a rare-cutter enzyme (Sbfl for instance) to increase read count per RAD tag per individual and reduce sequencing cost per individual [23-25]. Meanwhile, the QTL study using

Table 3 The enzyme sites comparison between Sbfl, BsaXI and AlfI

\begin{tabular}{|c|c|c|c|c|}
\hline Species & Size (G) & $S b f 1$ & $1 / 16^{\text {th }}$ of $B s a X I$ sites & $1 / 16^{\text {th }}$ of $A l f$ sites \\
\hline Arabidopsis thaliana & 0.12 & 627 & 2489 & 788 \\
\hline Cucumis sativus & 0.24 & 1197 & 4078 & 1485 \\
\hline Theobroma cacao & 0.33 & 1572 & 5113 & 2488 \\
\hline Oryza sativa & 0.37 & 5541 & 11303 & 5217 \\
\hline Vitis vinifera & 0.49 & 2594 & 9743 & 3879 \\
\hline Cajanus cajan & 0.61 & 1652 & 10145 & 3882 \\
\hline Solanum tuberosum & 0.73 & 2957 & 13256 & 4378 \\
\hline Sorghum bicolor & 0.74 & 11693 & 23749 & 9453 \\
\hline Glycine max & 0.97 & 3844 & 18121 & 8763 \\
\hline Zea mays & 2.06 & 50773 & 71938 & 27792 \\
\hline Average & 0.67 & 8245 & 16994 & 6812 \\
\hline Drosophila melanogaster & 0.17 & 2948 & 3637 & 3150 \\
\hline Apis mellifera & 0.23 & 551 & 3628 & 1327 \\
\hline Anopheles gambiae & 0.27 & 3164 & 6286 & 5009 \\
\hline Takifugu rubripes & 0.39 & 20718 & 13155 & 6496 \\
\hline Bombyx mori & 0.48 & 2041 & 6050 & 2958 \\
\hline Gallus gallus & 1.05 & 48010 & 28368 & 25022 \\
\hline Danio rerio & 1.41 & 30677 & 26637 & 21215 \\
\hline Mus musculus & 2.72 & 60302 & 83191 & 37944 \\
\hline Rattus norvegicus & 2.72 & 58283 & 78576 & 37232 \\
\hline Homo sapiens & 3.10 & 77095 & 80774 & 36471 \\
\hline Average & 1.17 & 30378 & 33030 & 17683 \\
\hline
\end{tabular}


Table 4 The scheme of how to apply I2b-RAD approach

\begin{tabular}{cccc}
\hline & High density genetic map & Common - lower density genetic map \\
\hline Application & Chromosome assembly & QTL mapping \\
Enzyme & BsaXI & BsaXI & Alfl \\
Adapter & Adapter1, adapter2 & Adapter1, adapter2 with & Adapter1, adapter2 with \\
& $5^{\prime}$-NNF-3' overhangs & $1 / 16^{\text {th }}$ & $1 / 16^{\text {th }}$ \\
Enzyme site percentage & $100 \%$ & SE50 of 384 samples; PE50 of & SE50 of 768 samples; \\
$\begin{array}{c}\text { The count of samples in a single } \\
\text { lane (Oryza sativa for instance) }\end{array}$ & SE50 of 24 samples; & PE50 of 48 samples; & PE50 of 1536 samples; \\
\hline
\end{tabular}

The count of samples by $1 / 16^{\text {th }}$ of Alf adapter were calculated based on the simulate data on Table 3 . The Alfl sites were nearly $1 / 2^{\text {th }}$ of the BsaXI in Oryza sativa.

ddRAD - sequencing permitted sequencing of over 1000 individuals in a single HiSeq 2000 lane [13]. The lower requirement of makers in QTL-research for 2b-RAD could be adjusted by two ways. One is to use Alfl. The density of Alfl sites was nearly $1 / 3^{\text {th }}$ in plants or $1 / 2^{\text {th }}$ in animals of BsaXI by simulation. The other way is to use the adapters with $5^{\prime}$-NNF-3' overhangs (F means a fixed base of anyone of $\mathrm{A}, \mathrm{T}, \mathrm{C}$ or $\mathrm{G})$ that targeted $1 / 16^{\mathrm{th}}\left(4^{4} /\right.$ $4^{6}$ ) of all BsaXI sites [14]. The digested fragment of Alf 1 has 2 bp random overhangs on the 3 ' ends. For modified adapters with $5^{\prime}$-NF-3' overhangs for Alfl, the targeted sites were also reduced to $1 / 16^{\text {th }}\left(4^{2} / 4^{4}\right)$. An addition comparison of the enzyme sites number was list on Table 3 . The result indicated that $1 / 16^{\text {th }}$ of Alf 1 sites was nearly the same sites of Sbfl in plants; while 1/ $16^{\text {th }}$ of BsaXI sites was nearly the same sites of Sbfl in animals. The adapters with the overhangs of "NNT" (T means any two kinds of bases of A, T, C or G) or "NNR" ( $\mathrm{R}$ means any three kinds of bases of $\mathrm{A}, \mathrm{T}, \mathrm{C}$ or $\mathrm{G}$ ) also could be used to increase markers than "NNF" if the marker density used by "NNF" adapters are fewer. So the adapter could be flexibly deployed of the fixed bases in its 3' overhangs according to marker density requirement, making $2 \mathrm{~b}-\mathrm{RAD}$ technique have multiple uses.

A detailed scheme of how to apply 2b-RAD approach in practice was showed on Table 4. Taking Oryza sativa for instance, sequencing of over 1000 individuals in a single HiSeq 2000 lane also can be achieved. Of course, genotyping of this approach can be used for natural population evolutional analysis.

\section{Conclusions}

By using barcodes adapters, 12 samples were pooled together as one sample to carry on following library procedure. It was not complicated for experiment operators to completing a library including 12 samples within 5 hours. Achieving 8 libraries containing 96 samples within one day wouldn't be a problem. The improvement of using barcodes adapters made 2b-RAD library preparation become sample and fast. Furthermore, the bioinformatics simulation and F2 population genotyping revealed that $2 \mathrm{~b}-\mathrm{RAD}$ data using BsaXI were effective for high density genetic map. More applications could be achieved by adjustment of enzyme and adapters. So an improved 2b-RAD genotyping approach was established in this research and named as I2b-RAD.

\section{Additional files}

Additional file 1: The adapters and PCR primers sequences.

Additional file 2: 2b-RAD protocol.

Additional file 3: The windows assembly simulation of BsaXI and EcoRI.

Additional file 4: The statistics of experiment reads.

Additional file 5: The statistics of reads mapping.

Additional file 6: The markers information derived from Stacks and SOAPsnp.

Additional file 7: The genetic maps information.

Competing interests

The authors declare that they have no competing interests.

\section{Authors' contributions}

GY conceived the improved 2b-RAD method, bioinformatics and wrote the paper. FD performed bioinformatics. SL planted rice population and extracted DNA with the assistance of LY, YJ and LZ. LY, WL and ZH modified the paper. $\mathrm{YH}, \mathrm{XX}$ and $\mathrm{ZH}$ provided supervisory roles. All authors have read and approved the final version of this manuscript.

\section{Acknowledgements}

We thank Miss Yan Ye for English revision and Mr. Chen Wang for assistance on figure drawing. This research was supported by the State Key Development Program for Basic Research of China_973 Program (NO.2011CB809202 and NO.2010CB125904), the Shenzhen Municipal Government of China (NO.JC201005260191A), the Shenzhen Key Laboratory of Transomics Biotechnologies (NO.CXB201108250096A).

Note: it is the responsibility of the author(s) to obtain permission from the copyright holder to reproduce figures or tables that have previously been published elsewhere. The figure and table in this paper were made by ourselves alone and were not referring to any copyright with others.

\section{Author details}

'Beijing Genome Institute-Shenzhen, Beishan Industrial Zone, Yantian District, Shenzhen 518083, China. ${ }^{2}$ Beijing Key Laboratory of Crop Genetic

Improvement, College of Agronomy and Biotechnology, China Agricultural University, Beijing 100193, China. ${ }^{3}$ National Institute of Biological Sciences, Beijing, Zhongguancun Life Science Park, Changping District, Beijing 100026, China.

Received: 6 November 2013 Accepted: 24 October 2014 Published: 5 November 2014 
References

1. Avise JC: Molecular Markers: Natural History and Evolution. New York: Chapman and Hall; 1994

2. Mohan M, Nair S, Bhagwat A, Krishna T, Yano M, Bhatia C, Sasaki T: Genome mapping, molecular markers and marker-assisted selection in crop plants. Mol Breed 1997, 3:87-103.

3. Sidransky D: Emerging molecular markers of cancer. Nat Rev Cancer 2002 2:210-219

4. Davey JW, Hohenlohe PA, Etter PD, Boone JQ, Catchen JM, Blaxter ML: Genome-wide genetic marker discovery and genotyping using nextgeneration sequencing. Nat Rev Genet 2011, 12:499-510.

5. Baird NA, Etter PD, Atwood TS, Currey MC, Shiver AL, Lewis ZA, Selker EU, Cresko WA, Johnson EA: Rapid SNP discovery and genetic mapping using sequenced RAD markers. PLoS One 2008, 3:e3376.

6. Bus A, Hecht J, Huettel B, Reinhardt R, Stich B: High-throughput polymorphism detection and genotyping in Brassica napus using next-generation RAD sequencing. BMC Genomics 2012, 13:281.

7. Liu MM, Davey JW, Banerjee R, Han J, Yang F, Aboobaker A, Blaxter ML, Davison A: Fine mapping of the pond snail left-right asymmetry (chirality) locus using RAD-Seq and fibre-FISH. PLOS One 2013, 8:e71067.

8. Wang X, Zhao L, Eaton D, Li D, Guo Z: Identification of SNP markers for inferring phylogeny in temperate bamboos (Poaceae: Bambusoideae) using RAD sequencing. Mol Ecol Resour 2013, 13:938-945.

9. Elshire RJ, Glaubitz JC, Sun Q, Poland JA, Kawamoto K, Buckler ES, Mitchell SE: A robust, simple genotyping-by-sequencing (GBS) approach for high diversity species. PLoS One 2011, 6:e19379.

10. Ward JA, Bhangoo J, Fernández-Fernández F, Moore P, Swanson J, Viola R, Velasco R, Bassil N, Weber CA, Sargent DJ: Saturated linkage map construction in Rubus idaeus using genotyping by sequencing and genome-independent imputation. BMC Genomics 2013, 14:2

11. Poland J, Endelman J, Dawson J, Rutkoski J, Wu S, Manes Y, Dreisigacker S, Crossa J, Sánchez-Villeda H, Sorrells M: Genomic selection in wheat breeding using genotyping-by-sequencing. The Plant Genome 2012, 5:103-113.

12. Poland JA, Brown PJ, Sorrells ME, Jannink J-L: Development of high-density genetic maps for barley and wheat using a novel two-enzyme genotyping-by-sequencing approach. PLoS One 2012, 7:e32253.

13. Peterson BK, Weber JN, Kay EH, Fisher HS, Hoekstra HE: Double digest RADseq: an inexpensive method for de novo SNP discovery and genotyping in model and non-model species. PLoS One 2012, 7:e37135.

14. Wang S, Meyer E, McKay JK, Matz MV: 2b-RAD: a simple and flexible method for genome-wide genotyping. Nat Methods 2012, 9:808-810.

15. Poland JA, Rife TW: Genotyping-by-sequencing for plant breeding and genetics. The Plant Genome 2012, 5:92-102.

16. Cronn R, Liston A, Parks M, Gernandt DS, Shen R, Mockler T: Multiplex sequencing of plant chloroplast genomes using Solexa sequencing-bysynthesis technology. Nucleic Acids Res 2008, 36:e122-e122.

17. Hamady M, Walker JJ, Harris JK, Gold NJ, Knight R: Error-correcting barcoded primers allow hundreds of samples to be pyrosequenced in multiplex. Nat Methods 2008, 5:235.

18. Smith AM, Heisler LE, Onge RPS, Farias-Hesson E, Wallace IM, Bodeau J, Harris AN, Perry KM, Giaever G, Pourmand N: Highly-multiplexed barcode sequencing: an efficient method for parallel analysis of pooled samples. Nucleic Acids Res 2010, 38:e142-e142.

19. Kircher M, Sawyer S, Meyer M: Double indexing overcomes inaccuracies in multiplex sequencing on the Illumina platform. Nucleic Acids Res 2012, 40:e3-e3.

20. Doyle JJ: A rapid DNA isolation procedure for small quantities of fresh leaf tissue. Phytochem Bull 1987, 19:11-15.

21. Chen H, Gong M, Liu T, Tian F, Wang J, Yu J, Zhang W, Zhang Y, Zhou Y: Dna tag and application thereof. PCT application NO: PCT/CN2011/079902; Publicaiton date: 2012.3.29

22. Catchen JM, Amores A, Hohenlohe P, Cresko W, Postlethwait JH: Stacks: building and genotyping loci de novo from short-read sequences. $G 3$ : Genes, Genomes 2011, 1:171-182.

23. Pfender W, Saha M, Johnson E, Slabaugh M: Mapping with RAD (restriction-site associated DNA) markers to rapidly identify QTL for stem rust resistance in Lolium perenne. Theor Appl Genet 2011, 122:1467-1480.

24. Chutimanitsakun Y, Nipper RW, Cuesta-Marcos A, Cistué L, Corey A, Filichkina T, Johnson EA, Hayes PM: Construction and application for QTL analysis of a Restriction Site Associated DNA (RAD) linkage map in barley. BMC Genomics $2011,12: 4$
25. Gonen S, Lowe NR, Cezard T, Gharbi K, Bishop SC, Houston RD: Linkage maps of the Atlantic salmon (Salmo salar) genome derived from RAD sequencing. BMC Genomics 2014, 15:166.

26. Yang H, Tao Y, Zheng Z, Li C, Sweetingham MW, Howieson JG: Application of next-generation sequencing for rapid marker development in molecular plant breeding: a case study on anthracnose disease resistance in Lupinus angustifolius L. BMC Genomics 2012, 13:318.

27. Kakioka R, Kokita T, Kumada H, Watanabe K, Okuda N: A RAD-based linkage map and comparative genomics in the gudgeons (genus Gnathopogon, Cyprinidae). BMC Genomics 2013, 14:32.

28. Consortium HG: Butterfly genome reveals promiscuous exchange of mimicry adaptations among species. Nature 2012, 487:94-98.

29. Zhang Q, Chen W, Sun L, Zhao F, Huang B, Yang W, Tao Y, Wang J, Yuan Z, Fan G: The genome of Prunus mume. Nat Commun 2012, 3:1318.

30. Willing E-M, Hoffmann M, Klein JD, Weigel D, Dreyer C: Paired-end RAD-seq for de novo assembly and marker design without available reference. Bioinformatics 2011, 27:2187-2193.

31. Baxter SW, Davey JW, Johnston JS, Shelton AM, Heckel DG, Jiggins CD, Blaxter ML: Linkage mapping and comparative genomics using next-generation RAD sequencing of a non-model organism. PLoS One 2011, 6(4):e19315.

doi:10.1186/1471-2164-15-956

Cite this article as: Guo et al:: An improved 2b-RAD approach (I2b-RAD) offering genotyping tested by a rice (Oryza sativa L.) F2 population. BMC Genomics 2014 15:956.

\section{Submit your next manuscript to BioMed Central and take full advantage of:}

- Convenient online submission

- Thorough peer review

- No space constraints or color figure charges

- Immediate publication on acceptance

- Inclusion in PubMed, CAS, Scopus and Google Scholar

- Research which is freely available for redistribution

Submit your manuscript at www.biomedcentral.com/submit
C Biomed Central 